\title{
Performance Analysis of CFAR Detection of Fluctuating Radar Targets in Nonideal Operating Environments
}

\author{
Mohame d B. El Mas hade
}

Electrical Engineering Dept., Faculty of Engineering, Al Azhar University, Nasr City, Cairo, Egypt

\begin{abstract}
A constant false alarm rate in the presence of variable levels of noise is usually a requirement placed on any modern radar. The CA- and OS-CFAR detectors are the most widely used ones in the CFAR world. The cell-averaging (CA) is the optimu m CFAR detector in terms of detection probability in ho mogeneous background when the reference cells have identical, independent and exponentially distributed signals. The ordered-statistic (OS) is an alternative to the CA processor, which trades a small loss in detection performance, relative to the CA scheme, in ideal conditions for much less performance degradation in non-ideal background environments. To benefice the merits of these well-known schemes, two modified versions (MX-\& MN-CFAR) have been recently suggested. This paper is devoted to the detection performance evaluation of these modified versions as well as a novel one (ML-CFAR). Exact formu las for their false alarm and detection performances are derived, in the absence as well as in the presence of spurious targets. The results of these performances obtained for Rayleigh clutter and Rayleigh target indicate that the MN-CFAR scheme performs nearly as good as OS detector in the presence of outlying targets and all the developed versions perform much better than that processor when the background environment is homogeneous. When compared to CA-CFAR, the modified schemes perform better in an ideal condition, and behave much better in the presence of interfering targets.
\end{abstract}

Keywords Adaptive Detection Techniques, Clutter Edge, Average Detection Threshold, Receiver Operating Characteristic, Target Mult iplicity Environments

\section{Introduction}

The detection of the radar's signal becomes a complex task when its returns are non-stationary background noise (or noise-plus-clutter). Many radar systems operate in an environment where the noise generated within its own receiver is not the dominant source of interference. Undesired echoes from rain and unwanted signals from other radiating sources often exceed the receiver noise level. These sources of interference may completely obliterate the radar display, or may overload the computer which is making Yes/No decisions as to which echoes are valid. To reduce this problem, radar detection processing can use an algorith $m$ to estimate the clutter energy in the target test cell and then adjust the detection threshold to reflect changes in this energy at different test cell positions. As the clutter-plus-noise power is not known a priori, a fixed threshold detection scheme cannot be applied to the radar return if the false alarm is to be controlled. In other words, the false alarm probability increases intolerably if a fixed threshold is used as a detection scheme. Therefore, adaptive

* Corresponding author:

ElMashade@yahoo.com (Mohamed B. El Mashade)

Published online at http://journal.sapub.org/ajsp

Copyright (C) 2012 Scientific \& Academic Publishing. All Rights Reserved threshold techniques are required in order to maintain a nearly constant false alarm rate. Because of the diversity of radar search environment, such as multiple targets and abrupt change in clutter, there exist no universal CFAR scheme. As a consequence, much attention has been paid to the task of designing and assessing these adaptive detection schemes $[1-3,8,11]$.

A variety of CFAR techniques are developed according to the logic used to es timate the unknown noise power level. An attractive class of such schemes, which set the threshold adaptively based on local information of total noise power, includes CA, OS and their modified versions. The threshold in a CFAR detector is set on a cell basis using estimated noise power by processing a group of reference cells surrounding the cell under investigation. The cell-averaging (CA) is an adaptive process or that can play an effective part in much noise and clutter environments, and provide nearly the best ability of signal detection while reserving the enough constant false alarm rate. This algorithm has the best performance in homogeneous background since it uses the maximum likelihood estimate of the noise power to set the adaptive threshold. However, the existence of heterogeneities in practical operating environments renders this processor ineffective[4-5,7,10]. Heterogeneities arise due to the presence of multiple targets and clutter edges. In the case of multiple targets, the detection probability of CA 
degrades seriously due to the non-avoidance of including the interfering signal power in noise level estimate. Consequently, this in turn leads to an unnecessary increase in overall threshold. When a clutter edge is present in the reference window and the test cell contains a clutter sample, a significant increase in the false alarm rate results. Both of these effects worsen as the clutter power increases. In order to overcome the problems as sociated with non-homogeneous noise backgrounds, alternative schemes have been developed to address this is sue, including ordered-statistic (OS) and its versions as well as various windowing techniques aimed to exclude heterogeneous regions . The well-known OS-CFAR process or estimates the noise power simply by selecting the $\mathrm{K}^{\text {th }}$ largest cell in the reference set of size N. It suffers only minor degradation in detection probability and can resolves closely spaced targets effectively for $\mathrm{K}$ different from the maximum. However, this processor is unable to prevent excessive false alarm rate at clutter edges, unless $\mathrm{K}$ is very close to $\mathrm{N}$, but in this case the processor suffers greater los $\mathrm{s}$ of detection performance[9,12].

In practical applications, the information about the number of interfering targets is not known in advance. The biggest samples of reference window are always trimmed with OS or TM method not only in multiple targets situation but also in homogeneous background. This, in turn, will result in additional CFAR loss, especially in the case where the size of the reference window is short. The resulting CFAR loss becomes unacceptable, and this can usually be encountered in complicated environment and lower SNR situation[13].

Two novel constant false alarm rate detectors; the maximum (MX)-CFAR and the minimum (MN)-CFAR, are recently appear in the literature[6]. These new formulas of CFAR detectors improve the conventional CA- and OS-CFAR schemes by making full use of the cell information. The novel CFAR processors combine the result of the CA and OS to get a better detection performance. In homogeneous background, the mathematical models of the two new CFA R detectors are derived and the performance of them has been evaluated and compared with that of CA- and OS-CFAR schemes. With the maximum-of CFAR detector, the problem of the increase of the false alarm probability due to the presence of a step discontinuity in the distributed clutter cloud has been treated. The minimum-of CFAR processor, on the other hand, was introduced to improve the resolution of closely spaced targets. These two new radar CFAR detectors synthesizing the advantages of CA- and OS-CFAR are analysed in an ideal environment. The modified CFAR structures make use of the two threshold settings of the well-known processors and compare them with the cell under test to achieve the judgment. For the MX-CFA Ralgorithm, when the cell under test is greater than both of the CA- and OS-CFAR thresholds, a target present will be declared. Otherwise, no target will be indicated. For the MN-CFAR algorithm, on the other hand, when the cell under test is greater than any of the two thresholds, a target present will be declared. Otherwise a no target declaration will be made.

Our goal in the present paper is to analyse these modified versions along with a new procedure in non-homogeneous situations. In section II, we formu late the underlined problem and describe the model of the processors under investigation. The performance of the schemes under consideration is analysed, in ideal (homogeneous) background environment, in section III. Section IV is devoted to the performance evaluation of these schemes in clutter edges and multitarget environments. In section $\mathrm{V}$, we present a brief discussion along with our conclusions.

\section{Model Description and Problem Formulation}

Fig.(1) depicts the architecture of the underlined CFAR detection procedure. It uses an adaptive threshold whose level is determined by the clutter and/or noise in the vicinity of the radar echo. Two tapped delay-lines sample echo signals from the environment in a number of reference cells located on both sides of the cell under test. The spacing between reference cells is equal to the radar range resolution (usually the pulse width). The output of the cell under investigation is the radar video output, which is compared to the adaptive threshold derived from the outputs of the tapped delay-lines. This threshold, therefore, represents the radar environment to either side of the tested cell. It changes as the radar environment changes and as the pulse travels out in time. When multiplied by a predetermined constant scale factor " $T$ ", it provides a detection threshold against which the content of the cell under investigation is compared to decide whether the radar target is present or absent. Thus, the constructed threshold can adapt to the environment as the pulse travels in time.

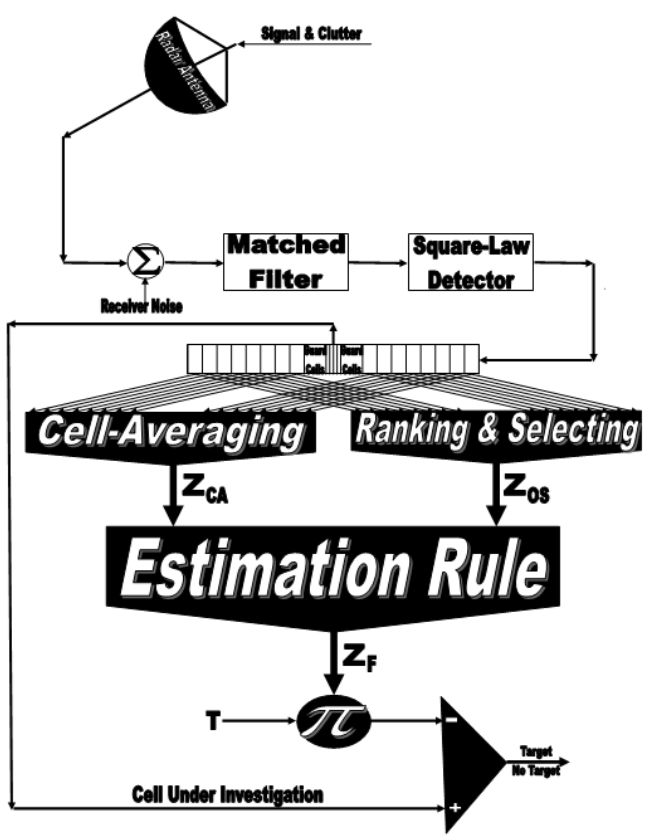

Figure 1. The diagrammatic sketch of the modified versions of adaptive radar target detectors 
Let the input target signal and noise to the square-law detector are represented by the complex vectors $\mathrm{u}+\mathrm{jv}$ and a $+\mathrm{jb}$, respectively. The variables "u" and " $\mathrm{v}$ " represent the in-phase and quadrature components of the target signal at the square-law detector, while the corresponding components of noise are denoted by "a" and "b", respectively. The target's signal is assumed to be independent of the noise and the in-phase samples are assumed to be independent and identically distributed (IID) with the Gaussian probability density function (PDF). The output of square-law detector, normalized to the noise power, is

$$
v \underline{\Delta} \frac{1}{2 \psi}\left(|u+a|^{2}+|v+b|^{2}\right)
$$

The characteristic function (CF) of " $v$ " can be expressed as

$C_{v}(\omega) \triangleq \int_{-\infty}^{\infty} p_{v}(y) \exp (-\omega y) d y$

$=\left\{\int_{-\infty}^{\infty} \int_{-\infty}^{\infty} p_{u}(u) p_{a}(a) \exp \left(-\frac{\omega}{2 \psi}|u+a|^{2}\right) d a d u\right\}^{2}$

In the above expression, $\mathrm{p}_{\mathrm{u}}(\mathrm{u})$ and $\mathrm{p}_{\mathrm{a}}(\mathrm{a})$ denote the PDF's of "u" and "a", respectively, " $\psi$ " represents the noise power. From our previous assumptions, one can write the joint PDF of "a" as

$$
p_{a}(a)=\frac{1}{\sqrt{2 \pi \psi}} \exp \left(-\frac{a^{2}}{2 \psi}\right)
$$

The substitution of Eq.(3) in Eq.(2) yields

$$
\begin{aligned}
& C_{v}(\omega)=\left\{\left(\frac{1}{2 \pi \psi}\right)^{1 / 2} \int_{-\infty}^{\infty} p_{u}(u) d u \int_{-\infty}^{\infty} \exp \left[-\frac{1}{2 \psi}\left(\omega|u+a|^{2}+|a|^{2}\right)\right] d a\right\}^{2} \\
& =\frac{1}{(\omega+1)}\left\{\int_{-\infty}^{\infty} p_{u}(u) \exp \left(-\frac{\omega}{2 \psi(\omega+1)}|u|^{2}\right) d u\right\}^{2}
\end{aligned}
$$

There are many PDF's for target cross section which are used to characterize fluctuating targets. The more important PDF is the so-called $\chi^{2}$ distribution with $2 \kappa$ degrees of freedom. The $\chi^{2}$ model approximates a target with a large reflector and a group of s mall reflectors, as well as a large reflector over a small range of aspect values. The $\chi^{2}$ family includes the Rayleigh (Swerling cases I \& II) model, the four-degree of freedom model (Swerling cases III \& IV), the Weinstock model $(\kappa<1)$ and the generalized model $(\kappa$ a positive real number)[7]. These models are used to represent complex targets such as aircraft and have the characteristic that the distribution is more concentrated about the mean as the value of the parameter $\kappa$ is increased. This distribution has a PDF given by[5]

$$
p(\sigma / \bar{\sigma})=\frac{1}{\Gamma(\kappa)}\left(\frac{\kappa}{\bar{\sigma}}\right)^{\kappa} \sigma^{\kappa-1} \exp \left(-\frac{\kappa}{\sigma} \sigma\right) U(\sigma)
$$

In the above expression, $\sigma$ represents the average cross section over all target fluctuations and $\mathrm{U}($.) denotes unit step function. When $\kappa=1$, the PDF of Eq.(5) reduces to the exponential or Rayleigh power distribution that applies to the Swerling case I. Swerling cases II, III, and IV correspond to $\kappa=M, 2$, and $2 \mathrm{M}$, respectively. Here, we are concerned only with single sweep case $(M=1)$.
It is finally of importance to note that the $\chi^{2}$ distribution with $2 \kappa$ degrees of freedom can be obtained by adding squared magnitude of $\kappa$ co mplex Gaussian random variables. In other words, if $\kappa=1$, then $\sigma$ may be generated as $\sigma=\mathrm{w}_{1}{ }^{2}+\mathrm{w}_{2}{ }^{2}$, where $\mathrm{w}_{\mathrm{i}}$ 's are IID Gaussian random variables, each with zero mean and $\bar{\sigma} / 2$ variance. The magnitude of the in-phase component $u\left(u=\left|w_{1}\right|\right)$ has a PDF given by

$$
p_{u}{ }^{(u) d u}=p_{w_{1}}\left(w_{1}\right) d w_{1}=\frac{1}{\sqrt{2 \pi \alpha}} \exp \left(-\frac{w_{1}^{2}}{2 \alpha}\right) d w_{1} \text { with } \alpha \underline{\Delta} \frac{\bar{\sigma}}{2}(6)
$$

The substitution of Eq.(6) into Eq.(4) y ields

$$
C_{v}(\omega)=\frac{1}{(\omega+1)}\left\{\int_{-\infty}^{\infty} \frac{1}{\sqrt{2 \pi \alpha}} \exp \left[-\frac{1}{2 \alpha}\left(1+\frac{\alpha}{\psi} \frac{\omega}{\omega+1}\right) w\right] d w\right\}^{2}(7)
$$

If we let the average signal-to-noise ratio (SNR) $\alpha / \psi=\mathrm{S}$ and after performing the specified integration, Eq.(7) takes the form:

$$
C_{v}(\omega)=\frac{1}{1+\psi(1+S) \omega}
$$

For the sake of simplicity, the above equation can be put in another clarified form as

$$
C_{v}(\omega)=\frac{D}{\omega+D} \text { with } D \triangleq \frac{1}{\psi(1+S)}
$$

The PDF of the output of the $i^{\text {th }}$ test tap is given by the Laplace inverse of Eq.(9), after making some minor modifications. If the $i^{\text {th }}$ test tap contains noise alone, we let $\mathrm{S}=0$, that is the average noise power at the receiver input is " $\psi$ ". If the $i^{\text {th }}$ range cell contains a return from the primary target, $\mathrm{Eq},(9)$ rests as it is where $\mathrm{S}$ represents the strength of the target return at the receiver input. On the other hand, if the $i^{\text {th }}$ test cell is corrupted by interfering target return, $\mathrm{S}$ must be changed to I, where I denotes the interference-to-noise (INR) at the receiver input. Finally, if the $\mathrm{i}^{\text {th }}$ cell is immersed in clutter, $\mathrm{S}$ is altered to $\mathrm{C}$ where $\mathrm{C}$ denotes clutter-to-noise ratio (CNR).

Implementing the generalized likelihood ratio test, the system decides the absence or the presence of a radar target according to whether

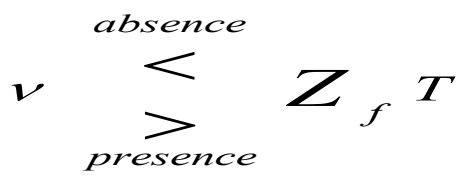

where $v$ denotes the content of the cell under test, $Z_{f}$ represents the final noise level estimate, and $\mathrm{T}$ is a constant scale factor used to achieve the required rate of false alarm.

Our approach in analysing a CFAR processor is to evaluate its probability of detection which is defined as

$$
P_{d} \Delta \operatorname{Pr}\left\{v>T Z_{f} \mid H_{1}\right\}=\operatorname{Pr}\left\{Z_{f}\langle v / T| H_{1}\right\}^{(11)}
$$

In terms of the PDF of $v$ and $Z_{\mathrm{f}}$, Eq. (11) can be formulated as

$$
P_{d}=\int_{0}^{\infty} p_{v}(v) \int_{0}^{v / T} p_{Z_{f}}(x) d x d v=\int_{0}^{\infty} p_{v}(v) F_{Z_{f}}(v / T) d v
$$

$\mathrm{Fz}_{\mathrm{f}}$ represents the cumulative distribution function (CDF) 
of $Z_{f}$. By taking the Laplace inverse of Eq.(9) and substituting it in the previous formula, one obtains

$$
P_{d_{d}}=\left(\frac{T}{\psi(1+S)}\right) \quad\left\{\Psi_{Z_{f}}(\omega)\right\} \mid \omega=\frac{T}{\psi(1+S)}
$$

$\Psi_{Z}$ (.) denotes the Laplace transformation of the CDF of the random variable $Z_{\mathrm{f}}$. The result of this analysis reveals that the Laplace transformation of the CDF of the final noise level estimate is the backbone of the CFAR performance evaluation. In terms of the $\mathrm{CF}$ of $Z_{\mathrm{f}}, \Psi_{Z}$ (.) can be calculated as

$$
\Psi_{Z_{f}}(\omega)=\frac{C_{Z_{f}}(\omega)}{\omega}
$$

Therefore, it is required now to derive the CF of the final noise level estimate for each one of the modified versions in order to analytically evaluate its performance either in the absence or in the presence of outliers. Finally, as $\mathrm{S}$ tends to zero $(\mathrm{S} \rightarrow 0), \mathrm{Eq},(13)$ leads to false alarm probability $\left(\mathrm{P}_{\mathrm{d}} \rightarrow\right.$ $\left.\mathrm{P}_{\mathrm{fa}}\right)$

\section{Performance of CFAR Detectors in ideal Situations}

The statistical model with un iform clutter background describes the classical signal situation with stationary noise in the reference window. In this model, two signal cases are of interest: (a) one target in the test cell in front of an otherwise uniform background, (b) uniform no ise situation throughout the reference window. In both cases, the noise in neighbourhood samples has a uniform statistic, i.e., the random variables $Y_{1}, Y_{2}, \ldots \ldots ., Y_{N}$ in the reference window are assumed to be statistically independent and identically distributed. In the absence of the target case, the random variable $v$ of the tested cell is assumed to be statistically independent of the neighbourhood and subject to the same distribution as the random variables $Y_{i}$ 's.

The ess ence of CFA R is to co mpare the decision statistic $v$ with an adaptive threshold $\mathrm{TZ}_{\mathrm{f}}$. The threshold coefficient $\mathrm{T}$ is a constant scale factor used to achieve the desired false alarm rate for a given window size $\mathrm{N}$ when the background noise is homogeneous. The statistic $Z_{\mathrm{f}}$ is a random variable whose distribution depends upon the particular CFAR scheme and the underlying distribution of each of the reference range samples.

As shown in the previous section, the characteristic function of the noise power level estimate ' $Z_{f}$ ' is the fundamental parameter that determines the processor performance either in homogeneous or non-homogeneous background environments. Therefore, our scope in the following subsections is to calculate this important parameter for the CFA R a gorith ms under consideration.

\subsection{Cell-Aver aging (CA) De tector}

A simple approach to achieve the CFAR condition is to set the detection threshold on the basis of the average noise power in a given number of reference cells where each of these cells is assumed to contain no targets. Such a scheme is denoted as cell-averaging (CA) CFAR processor. This detector is specifically tailored to provide good estimates of the noise power in the exponential PDF. Such estimation of the noise power is a sufficient statistic for the unknown noise power $\psi$ under the assumption of exponentially distributed homogeneous background. In other words, the noise power is estimated as

$$
Z_{C A} \quad \underline{1} \quad \frac{1}{N} \quad \sum_{i=1}^{N} Y_{i}
$$

Under the assumption that the surrounding range cells contain independent Gaussian noise samples with the same variance, $\mathrm{Z}_{\mathrm{CA}}$ is the maximum likelihood estimate of the common variance. In the absence of the radar target, each cell of the reference window has a CF of the same form as that of $v$ with the exception that A must be set to zero. Therefore, eliminating A in Eq.(9) leads to

$$
C_{Z_{C A}}(\omega)=\left(\frac{\theta}{\omega+\theta}\right)^{N} \text { with } \theta \triangleq \frac{N}{\psi}
$$

The rationale for the CA type of CFAR schemes is that by choosing the mean, the optimum CFAR processor in a homogeneous background when the reference cells contain IID observations governed by exponential distribution is achieved. As the size of the reference window increases, its detection performance approaches that of the optimum processor which is an algorithm based in its operation on a fixed threshold.

It is obviously of value to have some idea about the loss of detection power for a proposed CFAR scheme relative to the optimum processor for a homogeneous noise background. The simplest and more efficient method is that based on the average detection threshold (ADT) since the threshold and detection probability are closely related to each other. It is well known that as the threshold increases, the detection probability decreases accordingly and vice versa. Therefore, we use the concept of ADT to compare different CFAR processing techniques. Mathematically, this ADT is defined as[9]

$$
A D T \triangleq-\left.\frac{T}{\psi} \frac{d}{d \omega}\left\{C_{Z}(\varpi)\right\}\right|_{\omega=0}
$$

The substitution of Eq.(16) into Eq.(17) gives

$$
A D T_{C A}=T
$$

\subsection{Ordered-Statistic (OS) Detector}

The performance of CA-CFAR detector degrades rapidly in non-ideal conditions caused by multiple targets and nonuniform clutter. The ordered-statistic (OS) CFAR is an alternative to the CA processor. It trades a small loss in detection performance, relative to the CA scheme, in ideal situations for much less performance degradation in nonhomogeneous background environments.

Order statistics characterize amplitude information by ranking observations in which differently ranked outputs can 
estimate different statistical properties of the distribution from which they stem. The order statistic corresponding to a rank $\mathrm{K}$ is found by taking the set of $\mathrm{N}$ observations $\mathrm{Y}_{1}$, $\mathrm{Y}_{2}, \ldots \ldots ., \mathrm{Y}_{\mathrm{N}}$ and ordering them with respect to increasing magnitude in such a way that

$$
Y_{(1)} \leq Y_{(2)} \leq \ldots \leq Y_{(K-1)} \leq Y_{(K)} \leq Y_{(K+1)} \leq \ldots \leq Y_{(N)}
$$

$\mathrm{Y}_{(\mathrm{K})}$ represents the Kth order statistic. The central idea of OS-CFAR procedure is to select one certain value from the above sequence and to use it as an estimate $Z_{O S}$ for the average clutter power as observed in the reference window. Thus,

$$
Z_{O S}=Y_{(K)}, K \in\{1,2,3, \ldots, N\}
$$

We will denote by OS(K) the OS scheme with parameter $\mathrm{K}$. The value of $\mathrm{K}$ is generally chosen in such a way that the detection of radar target in homogeneous background environment is maximized.

In order to analyse the processor detection performance in uniform clutter background, the $\mathrm{CF}$ of the random variable $\mathrm{Z}_{\mathrm{OS}}$ is required. To evaluate this quantity, we start with the homogeneous representation of the CDF of the noise estimate which has a formula given by[12]

$$
F_{Z_{o s}}(z)=1-\sum_{\lambda=0}^{K-1}\left(\begin{array}{l}
N \\
\lambda
\end{array}\right)\left[1-F_{t}(z)\right]^{N-\lambda}\left\{F_{t}(z)\right\}^{\lambda}
$$

with

$$
F_{t}(z)=1-\exp \left(-\frac{z}{\psi}\right)
$$

By substituting Eq.(22) into Eq.(21) and taking the Laplace transformation, one obtains

$$
\Psi_{Z_{\text {Oos }}}(\omega)=\frac{1}{\omega}-\sum_{\lambda=0}^{K-1}\left(\begin{array}{l}
N \\
\lambda
\end{array}\right) \sum_{\ell=0}^{\lambda}\left(\begin{array}{l}
\lambda \\
\ell
\end{array}\right)(-1)^{\ell}\left(\omega+\frac{N+\ell-\lambda}{\psi}\right)^{-1}
$$
have

$$
A D T_{o S}=T \sum_{\lambda=0}^{K-1}\left(\begin{array}{l}
N \\
\lambda
\end{array}\right) \sum_{\ell=0}^{\lambda}\left(\begin{array}{l}
\lambda \\
\ell
\end{array}\right)(-1)^{\ell}\left(\frac{1}{N+\ell-\lambda}\right)
$$

The processor detection performance is easily evaluated once the $\mathrm{CF}$ of the noise level estimate $Z_{O S}$ is obtained. Finally, a desirable CFAR scheme would of course be one that is insensitive to changes in the total no ise power within the cells of the reference window so that a constant false alarm rate is maintained. This is actually the case of all architectures under consideration.

\subsection{Modified Versions}

Excessive numbers of false alarms in the CA processor at clutter edges and degradation of its detection performance in multiple target environments are the prime motivations for exploring other CFAR schemes. Since CA technique is the optimum CFAR processor given that the background is homogeneous and the reference cells contain independent and identically distributed observations governed by an exponential distribution, it is intuitive to use this identity in developing new versions. Additionally, the OS scheme has its immunity to the presence of interfering target returns amongst the contents of the reference window used for noise level estimation. For this important property of OS, it is of interest to include its basics in the development of the new CFAR schemes. Here, we are interesting in analysing three of such modified versions; namely; Mean-Level (ML), Maximu $\mathrm{m}(\mathrm{MX})$ and Minimu $\mathrm{m}(\mathrm{MN})$ operators.

\subsubsection{Mean-Level (ML)-CFAR}

The contents of the $\mathrm{N}$ reference samples feed two signal processors simultaneously. The first one of them estimates the unknown noise power emp loying the CA technique while the other does the same thing using OS basis. The two estimates are combined through the meal-level operation to estimate the final noise power level. Thus,

$$
Z_{M L} \triangleq \text { mean }\left(Z_{C A}, Z_{O S}\right)
$$

Where $Z_{\mathrm{CA}}$ and $Z_{\mathrm{OS}}$ are as previously given in Eqs. $(15,20)$, respectively. The $\omega$-domain representation of $Z_{f}$, which is $\mathrm{Z}_{\mathrm{ML}}$ in this case, is characterized by a CF of the form

$$
C_{Z_{M L}}(\omega)=C_{Z_{C A}}(\omega) C_{Z_{O S}}(\omega)=\omega C_{Z_{C A}}(\omega) \Psi_{O S}(\omega)(26)
$$

All the parameters of the above equation are previously evaluated as shown in Eqs,(14, 16, 23). Therefore, the processor detection performance is completely determined as Eq.(13) demonstrates. Finally, the ADT of this version of CFAR schemes can be easily computed and the result takes the form

$$
A D T_{M L}=A D T_{C A}+A D T_{O S}
$$

\subsubsection{Maximum (MX)-CFA R}

This version was specifically aimed at reducing the number of excessive false alarms at clutter edges. The final noise power is estimated fro $m$ the larger of the two separate noise level estimates computed for the CA and OS schemes. For this procedure of CFAR, we have

$$
Z_{M A X} \triangleq \max \left(Z_{C A}, Z_{O S}\right)
$$

In this case, $Z_{\mathrm{f}}$ has a $C D F$ given by $[10]$

$$
F_{Z_{M A X}}(z)=F_{Z_{C A}}(z) F_{Z_{O S}}(z)
$$

In order to analyse this version, the Laplace transformation of the above formula must be computed. To carry out this task, we start with Eq.(21) and use it in Eq.(28) which becomes

$$
\begin{aligned}
& F_{Z_{M A X}}(z)=\sum_{i=K}^{N}\left(\begin{array}{c}
N \\
i
\end{array}\right) \sum_{j=0}^{i}\left(\begin{array}{l}
i \\
j
\end{array}\right)(-1)^{j} \\
& \exp \left(-\frac{N+j-i}{\psi} z\right) F_{Z_{C A}}(z)
\end{aligned}
$$

By taking the Laplace transformation of Eq.(30), one obtains

$$
\Psi_{M A X}(\omega)=\sum_{i=K}^{N}\left(\begin{array}{c}
N \\
i
\end{array}\right) \sum_{j=0}^{i}\left(\begin{array}{c}
i \\
j
\end{array}\right)(-1)^{j} \Psi_{C A}(\omega) \mid \omega=\omega+\frac{N+j-i}{\psi}
$$

The substitution of $\mathrm{Eq},(31)$ into the definition of $\mathrm{P}_{\mathrm{d}}$ yields to the evaluation of the performance of the CFAR under investigation. On the other hand, the ADT of the underlined 
scheme is

$$
A D T_{M A X}=T \sum_{i=K}^{N}\left(\begin{array}{c}
N \\
i
\end{array}\right) \sum_{j=0}^{i}\left(\begin{array}{l}
i \\
j
\end{array}\right)(-1)^{j}\left(\frac{N}{2 N+j-i}\right)^{N+1}
$$

\subsubsection{Minimum (MN)-CFAR}

In order to prevent the suppression of closely spaced targets, the minimum version has been introduced. While testing for target presence at a particular range, the processor must not be influenced by the outlying target echoes. In MN-CFAR scheme, the noise power estimate is achieved by taking the smaller of $Z_{\mathrm{CA}}$ and $\mathrm{Z}_{\mathrm{OS}}$ as depicted in[5]. That is,

$$
Z_{\text {MIN }} \triangleq \min \left(Z_{C A}, Z_{O S}\right)
$$

In this case, the final noise level estimate has a CDF given by[12]

$$
F_{Z_{M I N}}(z)=F_{Z_{C A}}(z)+F_{Z_{O S}}(z)-F_{Z_{M A X}}(z)
$$

It is obvious from the above formula that there is a direct relation between the detection performance of MX-CFAR and that of MN-CFAR. This means that once the performance of MX operation is calculated, the performance of MN version is easily obtained. In $\omega$-doma in, Eq.(34) takes the form

$$
\Psi_{M I N}(\omega)=\Psi_{C A}(\omega)+\Psi_{O S}(\omega)-\Psi_{M A X}(\omega)
$$

Consequently, the ADT has a similar formu la as that given by Eq.(35). Thus, it is formulate $\mathrm{d}$ as

$$
A D T_{\text {MIN }}=A D T_{C A}+A D T_{o S}-A D T_{M A X}
$$

\subsection{Processor Performance Assess ment}

In this section, we give some representative numerical results, which will give an indication of the tightness of our previous analytical expressions. Since the performance of OS-CFAR is strongly dependent on the ranking para meter K, we choose the value that corresponds to the optimum detection performance in uniform noise background which is 21 for $\mathrm{N}=24[9]$.

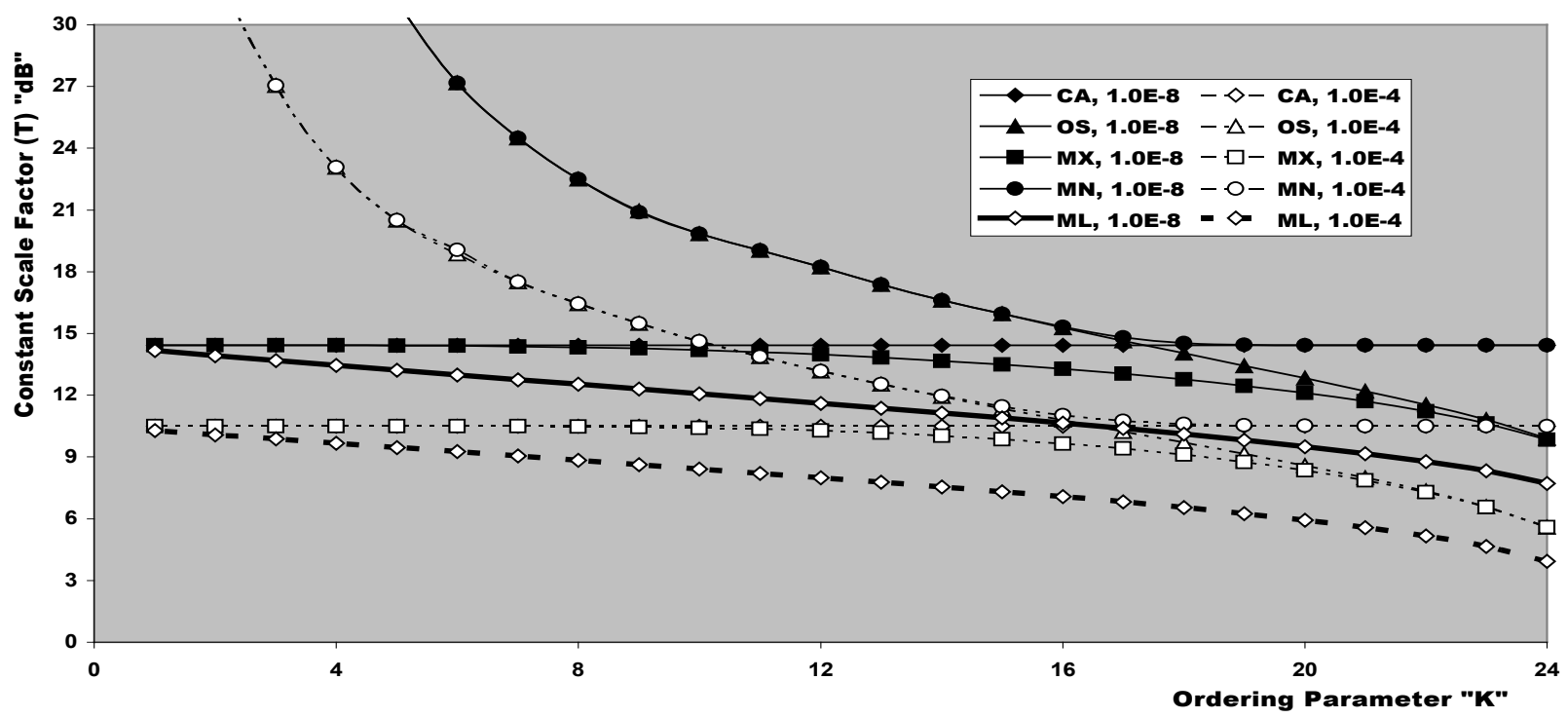

Figure 2. Thresholding constant as a function of the ordering parameter of CFAR algorithms when $N=24$

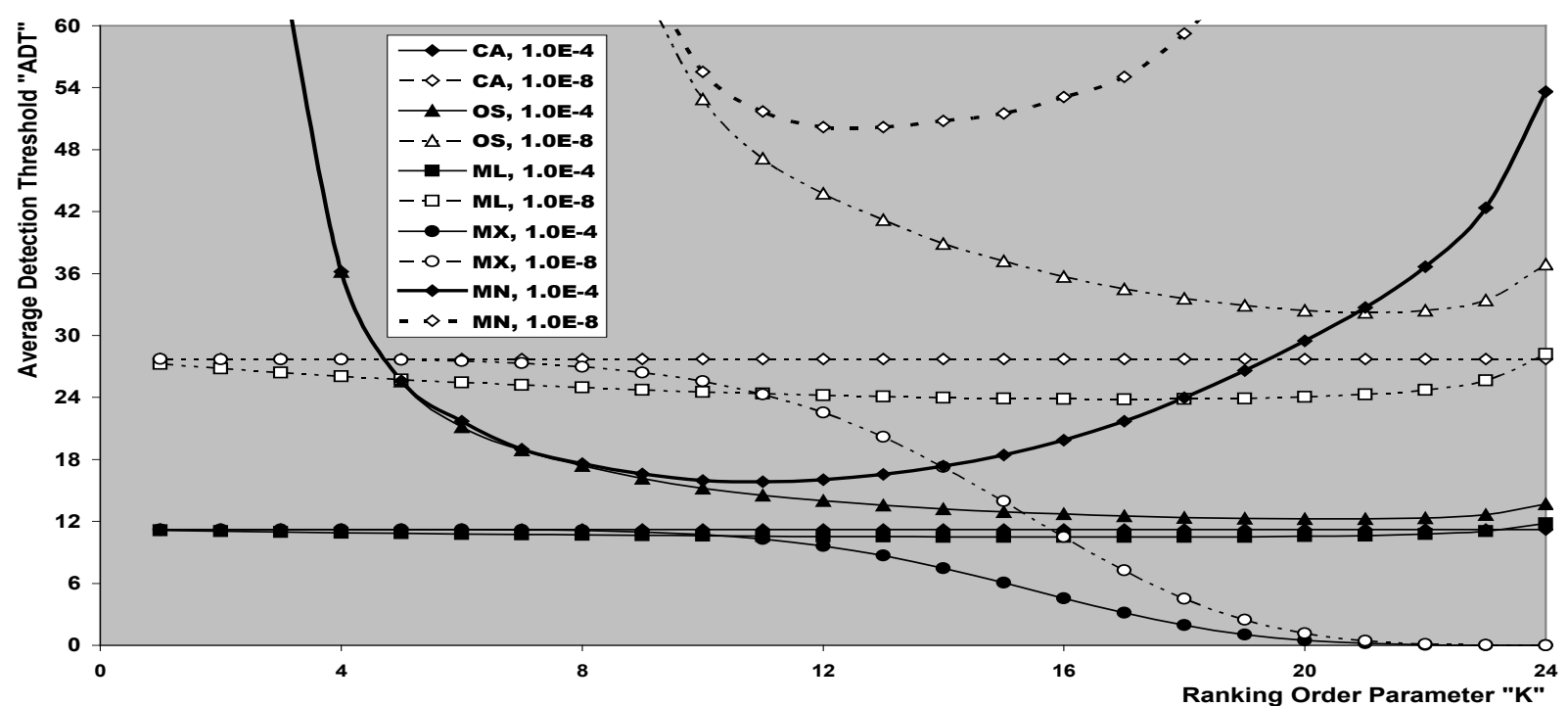

Figure 3. Average detection threshold(ADT)of CFAR processors as a function of ranking parameter $\mathrm{K}$ when $\mathrm{N}=24$ 


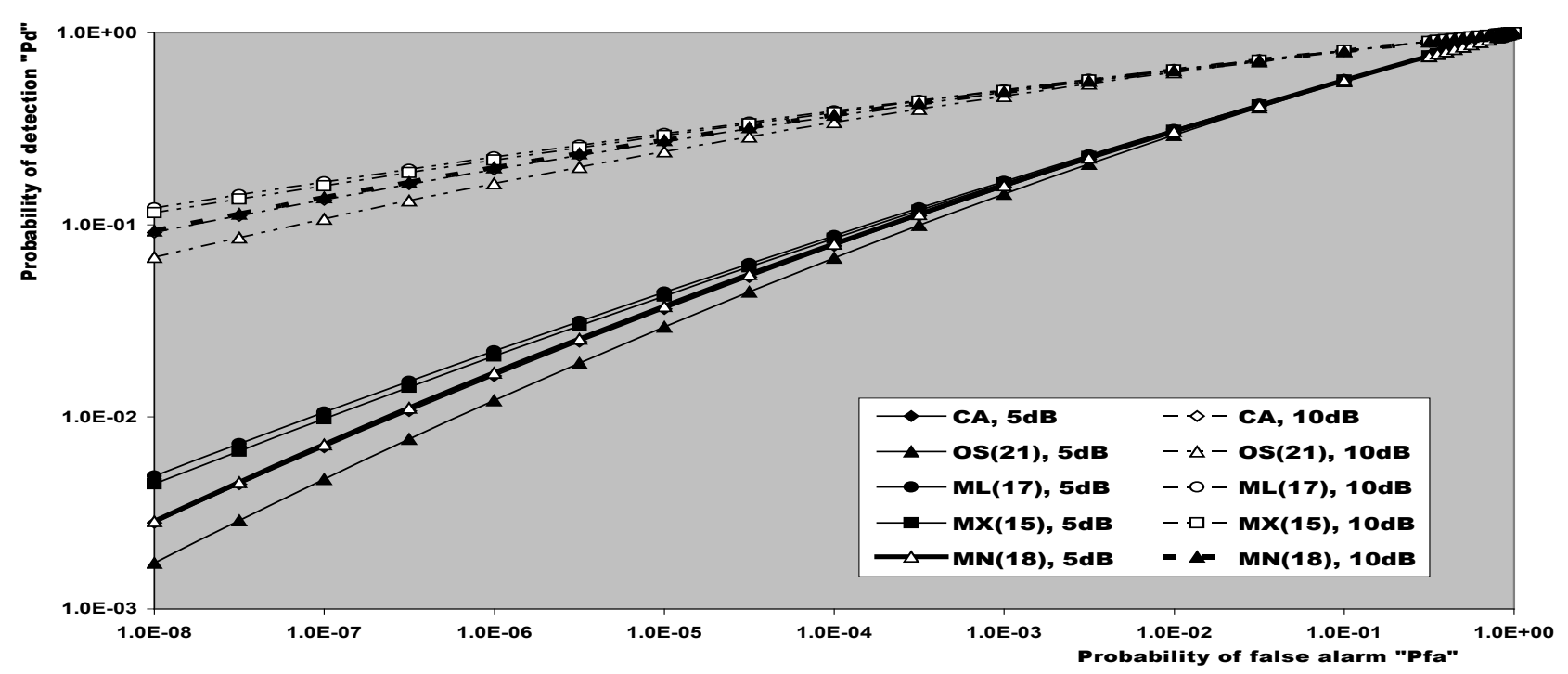

Figure 4. Receiver operat ing characterist ic (ROC) of CFAR processors in an ideal environment when $\mathrm{N}=24$

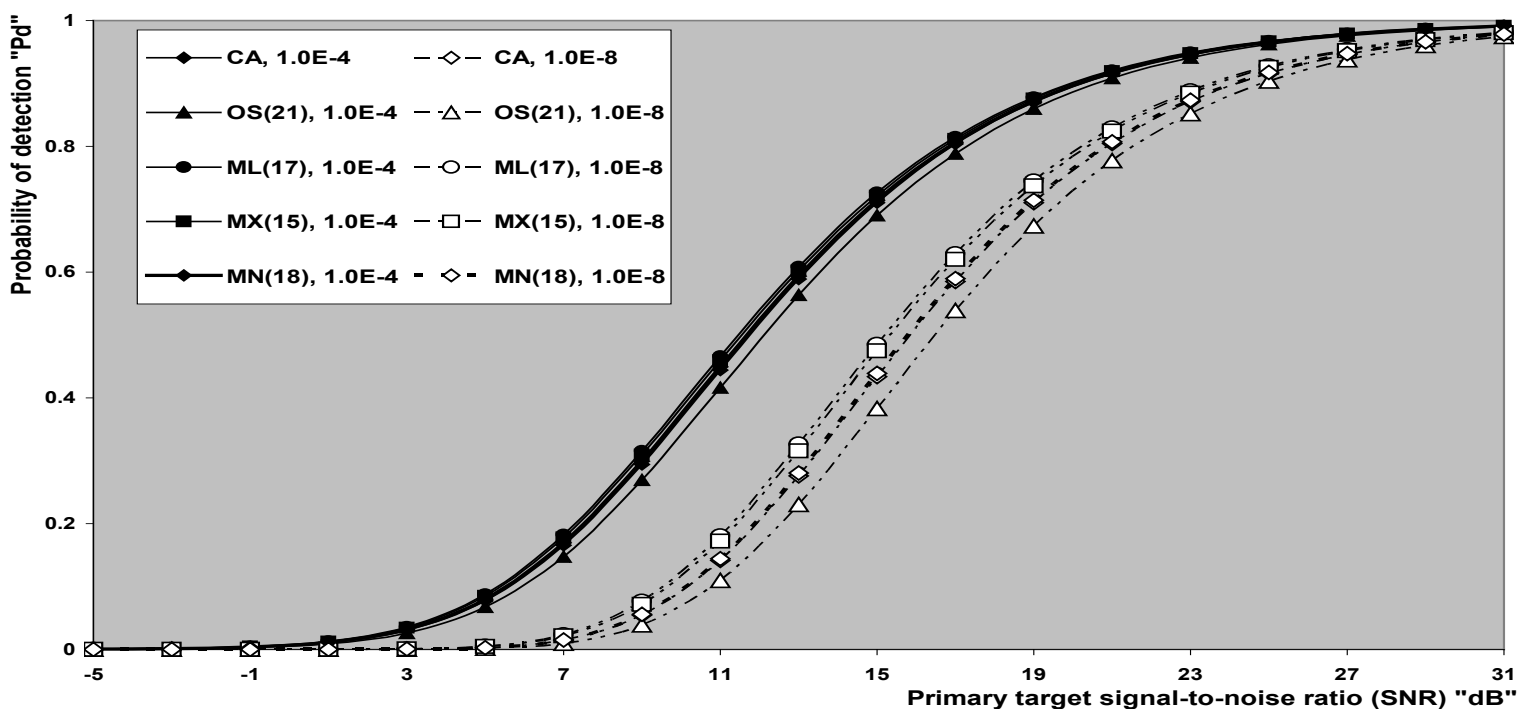

Figure 5. Single pulse detect ion performance of CFAR schemes in homogeneous situation for $\mathrm{N}=24$

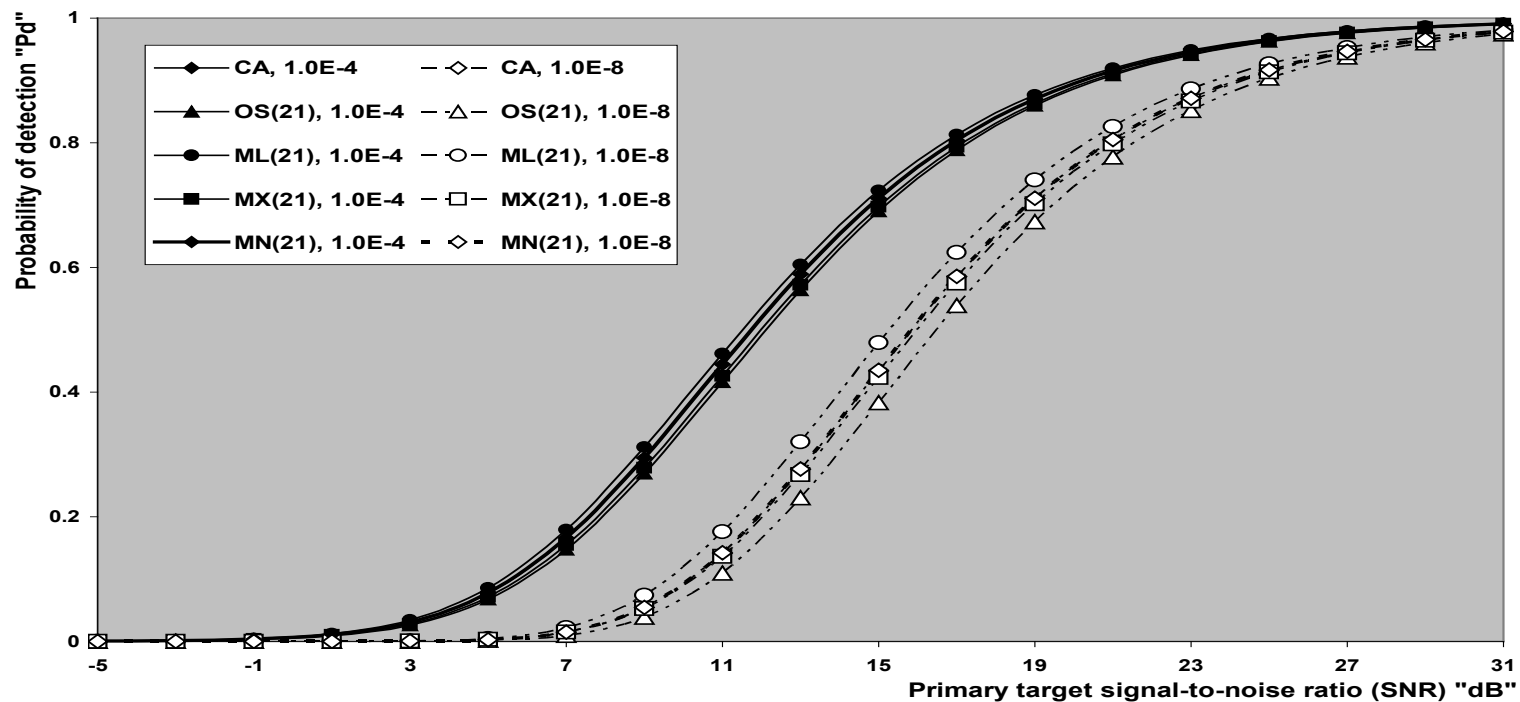

Figure 6. Single pulse detection performance of CFAR schemes with fixed value for the ranking order parameter "K" and operat ing in homogeneous environment when $\mathrm{N}-24$ 


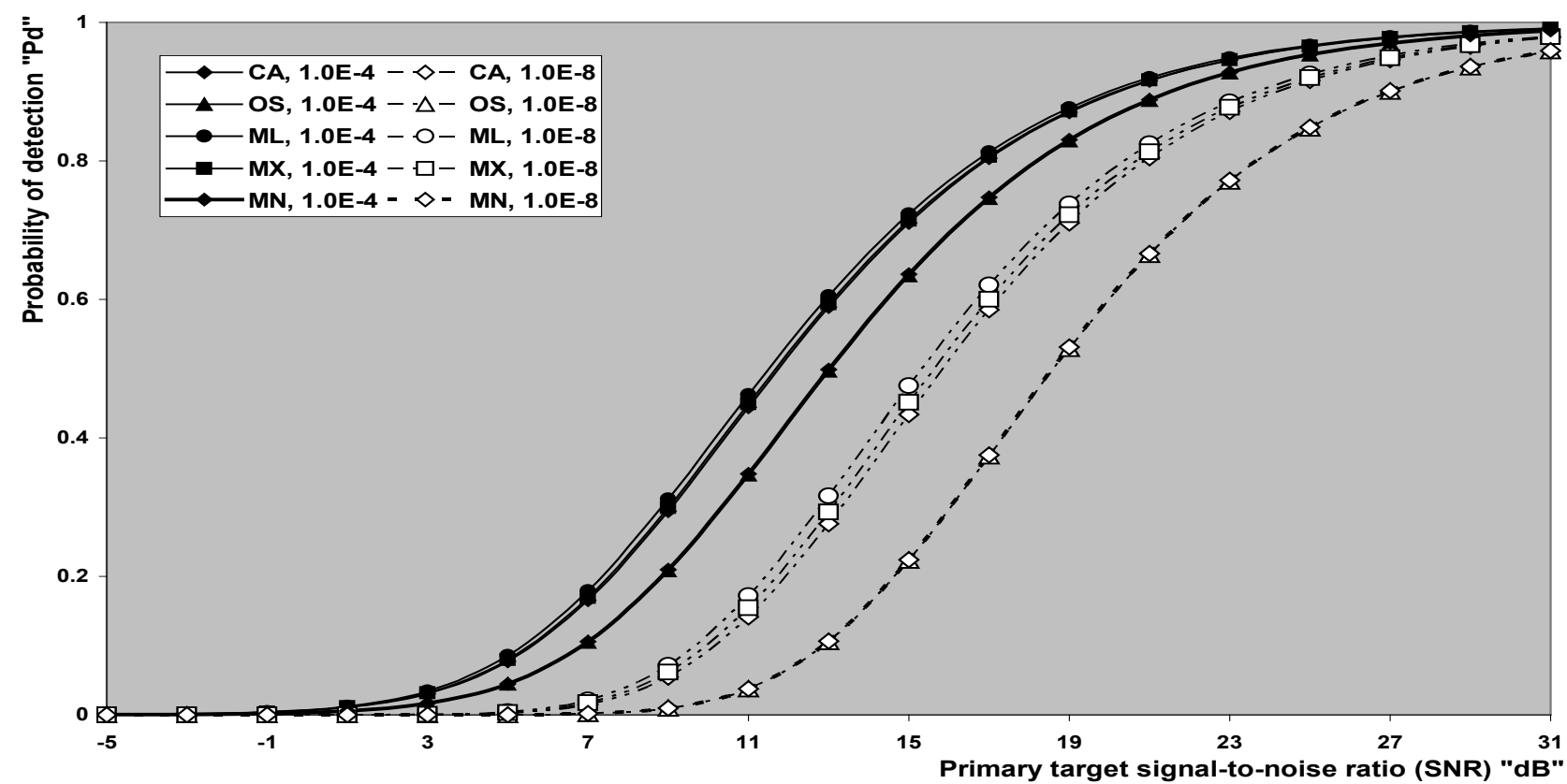

Figure 7. Single pulse detection performance of CFAR schemes with fixed "K" and operat ing in an ideal environment when N=24 and K=10

The actual probability of detection achieved by the modified versions of CFAR detectors in an ideal environment has been evaluated by means of computer simulation, where their derived analytical formulas are transferred into a sequence of programs using $\mathrm{C}++$ scientific language. The detection performances of the underlined detectors are also compared with that of CA- and OS-CFAR detectors to see to what extent the performance of the new versions improves.

Since the constant scale factor $\mathrm{T}$ represents the fundamental quantity in the evaluation of the processor performance, we start our presentation by running the program that concerns with the determination of $\mathrm{T}$ given that the false alarm probability is held fixed and the operating environment is free of interferers. The results of this program are depicted in Fig.(2) which illustrates the thresholding constant $\mathrm{T}$ as a function of the ranking order parameter $\mathrm{K}$ for $\mathrm{CA}, \mathrm{OS}$, and their modified versions. Two values for $\mathrm{P}_{\mathrm{fa}}\left(10^{-8}\right.$ $\& 10^{-4}$ ) are simulated. Since CA estimates its noise power by averaging the candidates of the reference set, the constant scale factor that this estimation must be multiplied, to achieve a prescribed rate of false alarm, becomes independent of $\mathrm{K}$. This is actually the case of our presentation. It is of importance to note that as $\mathrm{P}_{\mathrm{fa}}$ decreases, the detection threshold increases, given that the size of the reference set is held unchanged. The displayed results demonstrate this property for all the CFAR processors considered here. The behaviour of $\mathrm{T}$ for OS technique is as previously shown in the literature[9]. Since the MX version selects the maximum of the CA and OS noise level estimates, its noise power estimate equals that of $\mathrm{OS}$ for $\mathrm{K}=\mathrm{N}$, and consequently its scaling factor $\mathrm{T}$ coincides with that of $\mathrm{OS}$ at this value of $K$. As $K$ goes in the reverse direction $(K<N)$, there is a difference between the behaviour of $\mathrm{T}$ in the current case and that of OS state and this difference increases as $\mathrm{K}$ becomes smaller. At the limit, the thresholding constant $\mathrm{T}$ matches that of CA scheme. On the other hand, since the $\mathrm{MN}$ operation chooses the minimum of the two noise level estimates, its constant scale factor $\mathrm{T}$ approaches to that of $\mathrm{CA}$ technique for $\mathrm{K}=\mathrm{N}$. As $\mathrm{K}$ decreases, the $\mathrm{T}$ behaviour leaves that of CA and tends to be of OS scheme. Finally, the ML procedure sets its noise level estimate by summing that of CA and that of OS. This behaviour leads to increase the noise power estimate and consequently the scaling factor $T$ becomes smaller than the two original noise level estimates. As $\mathrm{K}$ moves towards the smaller values, $\mathrm{T}$ approaches to that of CA processor. In the limit, it coincides with the curve of $\mathrm{CA}$ scheme since $\mathrm{K}$ becomes negligible in comparis on with the ensemble average of the reference window. To illustrate this further, the ADT versus $\mathrm{K}$ at fixed $\mathrm{P}_{\mathrm{fa}}$ is plotted in Fig.(3) for the processing schemes under investigation. It is obvious that $\mathrm{ADT}=\mathrm{T}$ for the $\mathrm{CA}$ technique and hence it rests unchanged as $\mathrm{K}$ varies. On the other hand, the behaviour of ADT for the OS technique is as well known in the literature [12]. Since there is a direct proportionality between the ADT and T, the average detection threshold behaves as T with minor changes and this is common for all the CFAR presented in this paper.

Let us turn our attention to the receiver operating characteristics (ROC's) of the proces sors under consideration. This behaviour describes the variation of the detection probability as a function of the false a larm probability given that the SNR is held constant. Fig,(4) displays the results of these characteristics for the considered schemes for two values for the primary target SNR $(5 \& 10 \mathrm{~dB})$. The displayed results show that the processor detection performance improves, in a logarith mic linear fashion, as the rate of false alarm decreases and this is common for all the CFAR processors. It is well-known that the CA scheme is the optimum processor that has the highest detection 
performance in homogeneous situation. However, the performance of the modified versions outweighs that of the $\mathrm{CA}$ detector for some values for the ranking order parameter $\mathrm{K}$ as noted from the results of the figure under examination. The degree of superiority varies from one modified version to another. The ML operation has the top and the MN procedure has the lowest and this classification is verified for the two chosen values of SNR. To confirm this property, the detection probability as a function of SNR is drawn in Fig.(5) for $\mathrm{CA}, \mathrm{OS}$, and their modified versions for two fixed values of false alarm rate. It can be seen that the ML-CFAR achieves the highest detection performance and the MN-CFAR has the lowest degree of improvement. All the modified techniques give detection performance better than that of CA- and OS-CFAR schemes. Finally, we conclude that, the performance of the ML-CFAR is the best one among the five detectors, with MX-CFAR comes next to it, then MN-CFAR, after that CA-CFAR, and OS-CFA R gives the worst homogeneous detection performance in the selected group. This classification is obtained taking into account that each detector selects its ranking order parameter $\mathrm{K}$ that achieves its highest detection performance.

Now, let us show the influence of the ranking order parameter $\mathrm{K}$ on the homogeneous performance of the detectors under consideration. Figs.(6-7) demonstrate the effect of fixing $\mathrm{K}$ for all the schemes. In the first plot, $\mathrm{K}$ is taken as that corresponding to the maximum performance of OS $(K=21)$, while in the other figure, $\mathrm{K}$ is randomly chosen $(\mathrm{K}=10)$. The variation of the curves of Fig.(6) indicates that the ML version is still the king of the group and the process or OS has the worst detection performance. The behaviour of MN developing detector acts as the conventional CA scheme while the MX procedure gives lower performance than CA but still higher than that of OS processor. On the other hand, the results of Fig.(7) behave as those of the previous figure except that the gap between the family of CA and the family of OS becomes larger. The candidates of CA family include ML and MX modified versions, while the family of OS incorporates MN only. Additionally, it is obvious that as the false alarm decreases, the width of the gap between these families increases. Moreover, the MX developed processor gives higher detection performance than $\mathrm{CA}$ for this value of the ranking order parameter $\mathrm{K}(\mathrm{K}=10)$.

\section{Performance of CFAR Schemes in Non-homogeneous Environments}

The CFAR algorithms were originally developed using a statistical model of uniform background noise. However, this is not representative of real situations. It is impossible to describe all radar working conditions by a single model, yet consideration of a larger nu mber of different situations might be confusing. For these reasons, three different signal situations are selected: uniform clutter, clutter edges and multip le targets. The performance of the CFAR algorithms for uniform clutter model was completely evaluated in the previous section. Clutter edges, on the other hand, are used to describe transition areas between regions with very different noise characteristics [8]. This situation occurs when the total noise power received within a single reference window changes abruptly. The presence of such a clutter edge may result in severe performance degradation in an adaptive thres hold scheme leading to excessive false a larms or serious target masking depending upon whether the cell under test is a sample from clutter background or from relatively clear background with target return, respectively. On the other hand, multiple target situations occur occasionally in radar signal processing when two or more targets are at a very similar range. The consequent masking of one target by the others is called suppression. These interferers can arise from either real object returns or pulsed noise jamming. From a statistical point of $v$ iew, this implies that the reference samples, although still independent of one another, are no longer identically distributed.

\subsection{Cell-Aver aging (CA) De tector}

In order to analyse the CFAR detection performance when the candidates of the reference window no longer contain radar returns fro m a ho mogeneous background, as in the case of clutter edges, the as sumption of statistical independence of the reference cells is retained. Let us assume that the reference set contains $\mathrm{R}$ cells from clutter background with noise power $\psi(1+\mathbf{C})$, with $\mathbf{C}$ denotes the clutter-to-thermal noise ratio (CNR), and $\mathrm{N}$-R cells fro mclear background with noise power " $\psi$ ". Thus, the estimated total noise power level is obtained from

$$
Z_{C A}=\frac{1}{N}\left(\sum_{\lambda=1}^{R} Y_{\lambda}+\sum_{\ell=R+1}^{N} Y_{\ell}\right) \triangleq Z_{C}+Z_{c}
$$

The random variable $Z_{C}$ rep resenting the clutter return and that denoting the clear background $Z_{c}$ have $C F$ 's given by a similar form as that indicated in Eq.(16) after minor changing of its parameters. Since the candidates of each type are assumed to be statistically independent, we have

$$
C_{Z_{C}}(\omega)=\left(\frac{\frac{N}{\psi(1+C)}}{\omega+\frac{N}{\psi(1+C)}}\right)^{R} \& C_{Z_{c}}(\omega)=\left(\frac{\frac{N}{\psi}}{\omega+\frac{N}{\psi}}\right)^{N-R}
$$

Since $Z_{C}$ and $Z_{c}$ are statistically independent, the $C F$ of $Z_{C A}$ is simply the product of the individual CF's of $Z_{C}$ and $Z_{c}$. Therefore, assuming that the cell under investigation is from clear background, the false alarm probability has a formula given by

$$
P_{f a}=\left.\frac{T}{\psi} \Psi_{C A}(\omega)\right|_{\omega=\frac{T}{\psi}} \& \Psi_{C A}(\omega)=\frac{1}{\omega} C_{Z_{C}}(\omega) C_{Z_{c}}(\omega)
$$

As the window sweeps over the range cells, more cells from clutter background enter into the reference window. When the cell under test comes fro m clutter background, the probability of false alarm takes the form; 


$$
P_{f a}=\left.\frac{T}{\psi(1+C)} \Psi_{C A}(\omega)\right|_{\omega=\frac{T}{\psi(1+C)}}
$$

Another situation of non-homogeneity is the case of multip le targets. In our analys is and study of this situation for which the reference cells don't follow a single common PDF, we are concerned with increases in the value of $\psi$ for some isolated reference cells due to the presence of secondary targets. The amplitudes of all the targets present amongst the candidates of the reference window are assumed to be of the same strength and to fluctuate in accordance with SWII fluctuation model as the primary target. The interference-to-noise ratio (INR) for each of the spurious targets is taken as a common parameter and is denoted by $\mathbf{I}$. Thus, for reference cells containing extraneous target returns, the total background noise power is $\psi(1+\mathbf{I})$, while the remaining reference cells have identical noise power of $\psi$ value. The determination of the detection probability is similar to the one presented above for the clutter power transitions with some changes in the definition of the parameters. R will now represent the number of outlying target returns amongst the contents of the reference set.

\subsection{Or dered-Statistic (OS) Detector}

In order to analyse the processor performance in non-homogeneous background, we follow the same steps as those presented for CA technique. Consider the same previously stated situation where there are $\mathrm{R}$ reference samples contaminated by clutter returns, each with power level $\psi(1+\mathrm{C})$, and the remaining $\mathrm{N}-\mathrm{R}$ reference cells contain thermal noise only with power level $\psi$. Under these assumptions, the $\mathrm{K}^{\text {th }}$ ordered sample, which represents the noise power level estimate in the OS detector, has a CDF given by[13]

$$
\begin{aligned}
F_{Z_{O S}}(z)= & \sum_{i=K}^{N} \sum_{j=\max (0, i-R)}^{\min (i, N-R)}\left(\begin{array}{c}
N-R \\
j
\end{array}\right)\left(\begin{array}{c}
R \\
i-j
\end{array}\right)\left[1-F_{c}(z)\right]^{N-R-j} \\
& \left\{F_{c}(z)\right\}^{j}\left[1-F_{C}(z)\right]^{R-i+j}\left\{F_{C}(z)\right\}^{i-j}
\end{aligned}
$$

$\mathrm{F}_{\mathrm{c}}($.$) denotes the \mathrm{CDF}$ of the reference cell that contains a clear background and $F_{C}($.) denotes the same thing for the reference cell that belongs to clutter. Mathematically, these functions can be formulated as:

$$
F_{c}(z)=1-\exp \left(-\frac{z}{\psi}\right) \& F_{C}(z)=1-\exp \left(-\frac{z}{\psi(1+C)}\right)
$$

By substituting Eq.(42) into Eq.(41) and taking the Laplace transformation of its resulting formula, one obtains

$$
\begin{aligned}
& \Psi_{O S}(\omega)=\sum_{i=K}^{N} \sum_{j=\max (0, i-R)}^{\min (i, N-R)}\left(\begin{array}{c}
N-R \\
j
\end{array}\right)\left(\begin{array}{c}
R \\
i-j
\end{array}\right) \sum_{\lambda=0}^{j}\left(\begin{array}{l}
j \\
\lambda
\end{array}\right) \\
& (-1)^{\lambda} \sum_{\ell=0}^{i-j}\left(\begin{array}{c}
i-j \\
\ell
\end{array}\right)(-1)^{\ell}\left(\omega+\frac{N-R-j+\lambda}{\psi}+\frac{R-i+j+\ell}{\psi(1+C)}\right)^{-1}
\end{aligned}
$$

Once the $\omega$-do main representation of the CDF of the noise power level estimate is calculated, the processor performance evaluation becomes an easy task, as we have previously shown, where the false alarm and detection probabilities are completely dependent on this trans formation.

In the presence of interferers, the OS-CFAR processor performance is highly dependent upon the value of K. For example, if a single extraneous target appears in the reference window of appreciable magnitude, it occupies the highest ranked cell with high probability. If $K$ is chosen to be $\mathrm{N}$, the estimate will almost always set the threshold based on the value of interfering target. This increases the overall threshold and may lead to a target miss. If, on the other hand, $\mathrm{K}$ is chosen to be less than the maximum value, the OS-CFAR scheme will be influenced only slightly for up to $\mathrm{N}-\mathrm{K}$ spurious targets.

\subsection{Modified Versions}

\subsubsection{Mean-Level(ML)-CFAR}

In this type of modified versions, the noise level estimate employing CA technique and that using OS procedure are combined through the mean-level operation to obtain the final noise power level estimate. The purpose of this processing is to improve the processor performance in the absence as well as in the presence of outlying targets. In the case where the operating environment has a clutter edge, the evaluation of the processor false alarm rate follows the same procedure as in CA and OS cases. Using Eqs. $(39,14,43)$ in Eq.(26), the Lap lace transformation of the CDF of the noise level estimate of this processor can be computed and consequently, the evaluation of false alarm rate in clutter edges and the detection performance in multiple-target situations are completely determined.

\subsubsection{Maximum (MX)-CFA R}

As previously stated, for MX-CFAR, the content of the cell under test must be greater than both the CA-CFAR threshold and the OS-CFAR threshold to declare the presence of a target, which is equivalent to choosing the maximum value of the CA-CFAR and the OS-CFAR thresholds and compare it with the target cell (cell under investigation). For the processor performance to be evaluated, let us go to calculate the Laplace transformation of the CDF of the final noise power level estimate of the underlined detector. By substituting Eq.(41) in Eq.(29) and transferring it to the $\omega$-do main representation, we have

$$
\begin{aligned}
& \Psi_{M X}(\omega)=\sum_{i=K}^{N} \sum_{j=\max (0, i-R)}^{\min (i, N-R)}\left(\begin{array}{c}
N-R \\
j
\end{array}\right)\left(\begin{array}{c}
R \\
i-j
\end{array}\right) \sum_{\lambda=0}^{j}\left(\begin{array}{l}
j \\
\lambda
\end{array}\right)(-1)^{\lambda} \\
& \sum_{\ell=0}^{i-j}\left(\begin{array}{c}
i-j \\
\ell
\end{array}\right)(-1)^{\ell} \Psi_{C A}(\omega) \mid \begin{array}{l}
\omega=\omega+\frac{N-R-j+\lambda}{\psi}+\frac{R-i+j+\ell}{\psi(1+C)}
\end{array}
\end{aligned}
$$

Again, once the Laplace transformation of the CDF of the noise level estimate is achieved, the false alarm rate performance in the presence of clutter edges and the detection probability in the presence of spurious targets are completely known since $\Psi_{\mathrm{MX}}(\omega)$ represents the backbone of their evaluation. 


\subsubsection{Minimum (MN)-CFAR}

As the case of MN-CFAR, the content of the target cell should be greater than the CA-CFAR threshold or the OS-CFAR threshold to declare a target present, which is equivalent to choosing the minimu m value of the CA-CFAR and the OS-CFAR thresholds and compare it with the primary target return to indicate its presence. As Eq.(35) demonstrates, there is a direct relation between the performance evaluation of this processor and that of the MX-CFAR scheme. This means that once the false alarm rate probability in clutter edges situation and the detection probability in multiple target environments are calculated for the MX-CFAR version, their evaluations in the case of MN-CFA R detector are automatically known given that the performances of the original processors (CA and OS) are completely determined. This is actually the case of our treatment.

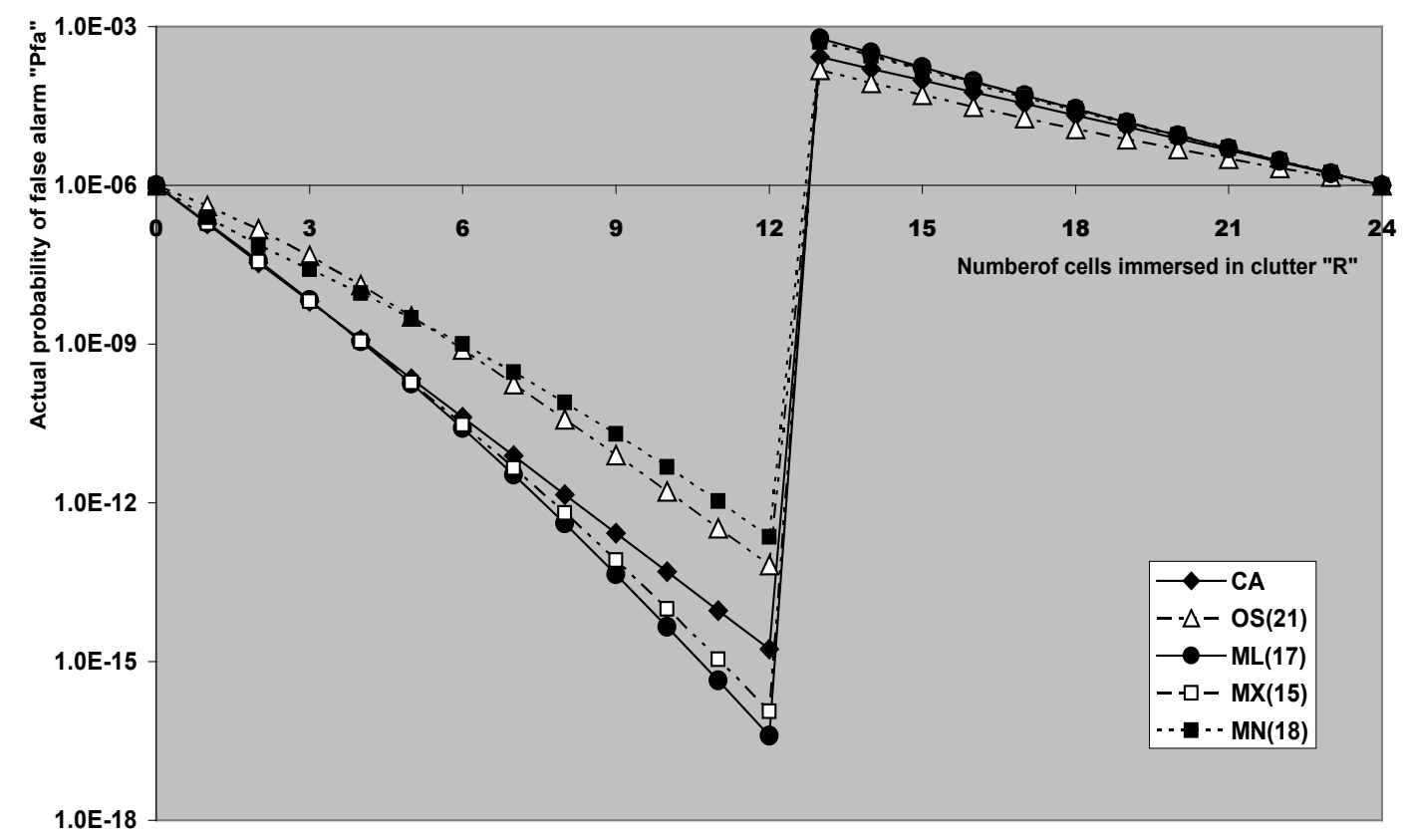

Figure 8. False alarm rate performance of CFAR schemes in the presence of clutter edges with $\mathrm{ONR}=10 \mathrm{~dB}$ for $\mathrm{N}=24$, and design $\mathrm{P}$ fa $=1.0 \mathrm{E}-6$

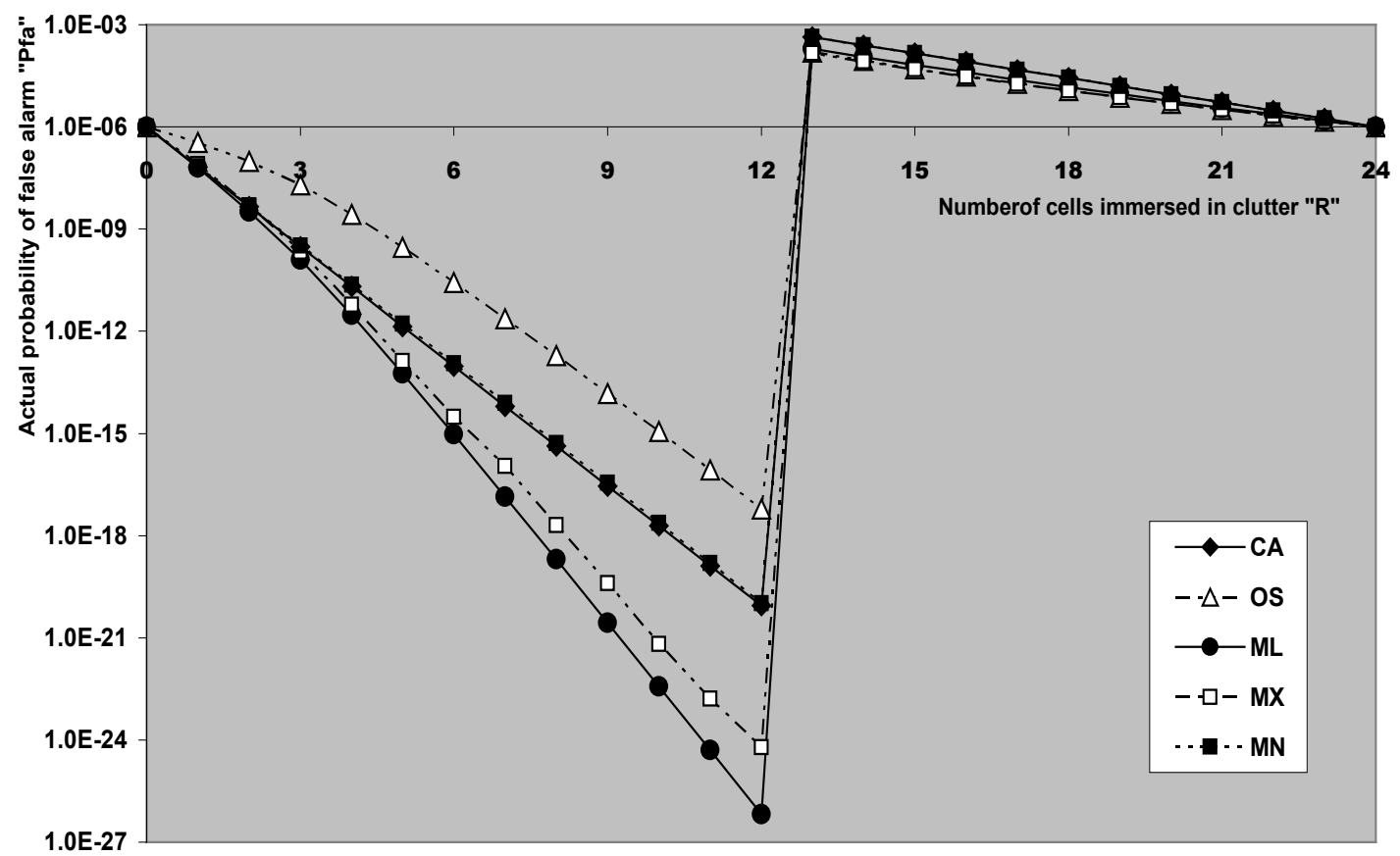

Figure 9. False alarm rate performance of $C F A R$ schemes operating in clutter edge environment with $C N R=15 \mathrm{~dB}$ for $\mathrm{N}=24, \mathrm{~K}=21$, and design $\mathrm{Pfa}=1.0 \mathrm{E}-6$ 


\subsection{Process or Perfor mance Ass ess ment}

Here, we provide a variety of numerical results for the performance of CA- and OS-CFAR processors as well as their modified versions in the case where the operating environment contains either clutter edge or a number of outlying targets. Since the optimum value of $\mathrm{K}$, for a reference window of size 24 , is 21 , we have assumed that there are three interfering target returns amongst the contents of the estimation cells. This value of extraneous target returns is the maximum allowable value before the OS performance degradation occurs. Our results are obtained for a possible practical situation where the primary and the secondary interfering targets fluctuate in accordance SWII model and of equal target return strength ( $\mathrm{INR}=\mathrm{SNR})$. The design probability of false alarm is maintained at its previous value $\left(\mathrm{P}_{\mathrm{fa}}=10^{-6}\right)$. The false alarm performance of the underlined CFAR processors when the reference window contains a clutter edge is numerically evaluated. Fig.(8) illustrates the actual value of false alarm rate in the presence of clutter edge when there are $\mathrm{R}$ cells immersed in clutter. The result in this figure is obtained for a clutter to noise ratio (CNR) of $10 \mathrm{~dB}$ and for the design value of the ranking order parameter $\mathrm{K}$ for each scheme. The displayed results show that the $\operatorname{OS}(21)$ has the lowest false alarm rate at clutter edge and is followed by $\mathrm{CA}, \mathrm{MN}(18), \operatorname{MX}(15)$, and $\operatorname{ML}(17)$ comes the last one in the concerned group. In other words, OS(21) has the best performance among all the underlined CFAR processors in the presence of non-uniform clutter. To show the effect of changing $\mathrm{K}$ on the processor performance, Fig.(9) illustrates the same thing as the previous figure except that $\mathrm{K}$ is held fixed $(\mathrm{K}=21)$ for all the schemes and the CNR is changed to $15 \mathrm{~dB}$. In this situation, the MX version gives the best false alarm rate and the OS comes next. The $\mathrm{CA}$ and $\mathrm{MN}$ have the worst rate of false alarm under these circumstances. Therefore, the selection of the ranking order parameter $\mathrm{K}$ plays an important rule in the reaction of the process or to the environmental conditions.

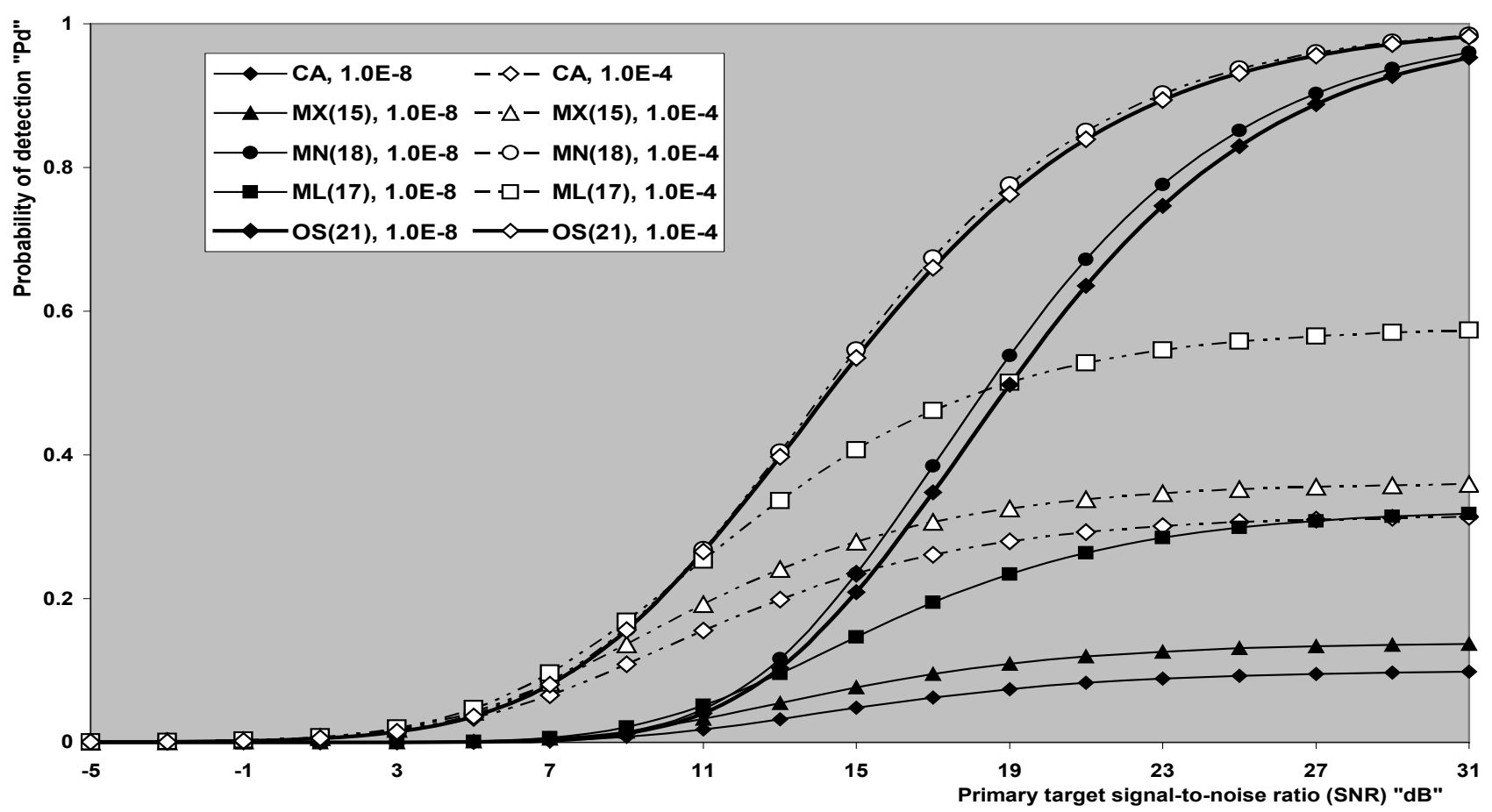

Figure 10. Single pulse multiple target detection performance of CFAR schemes for CFAR schemes for $N=24$, and $R=3$ 


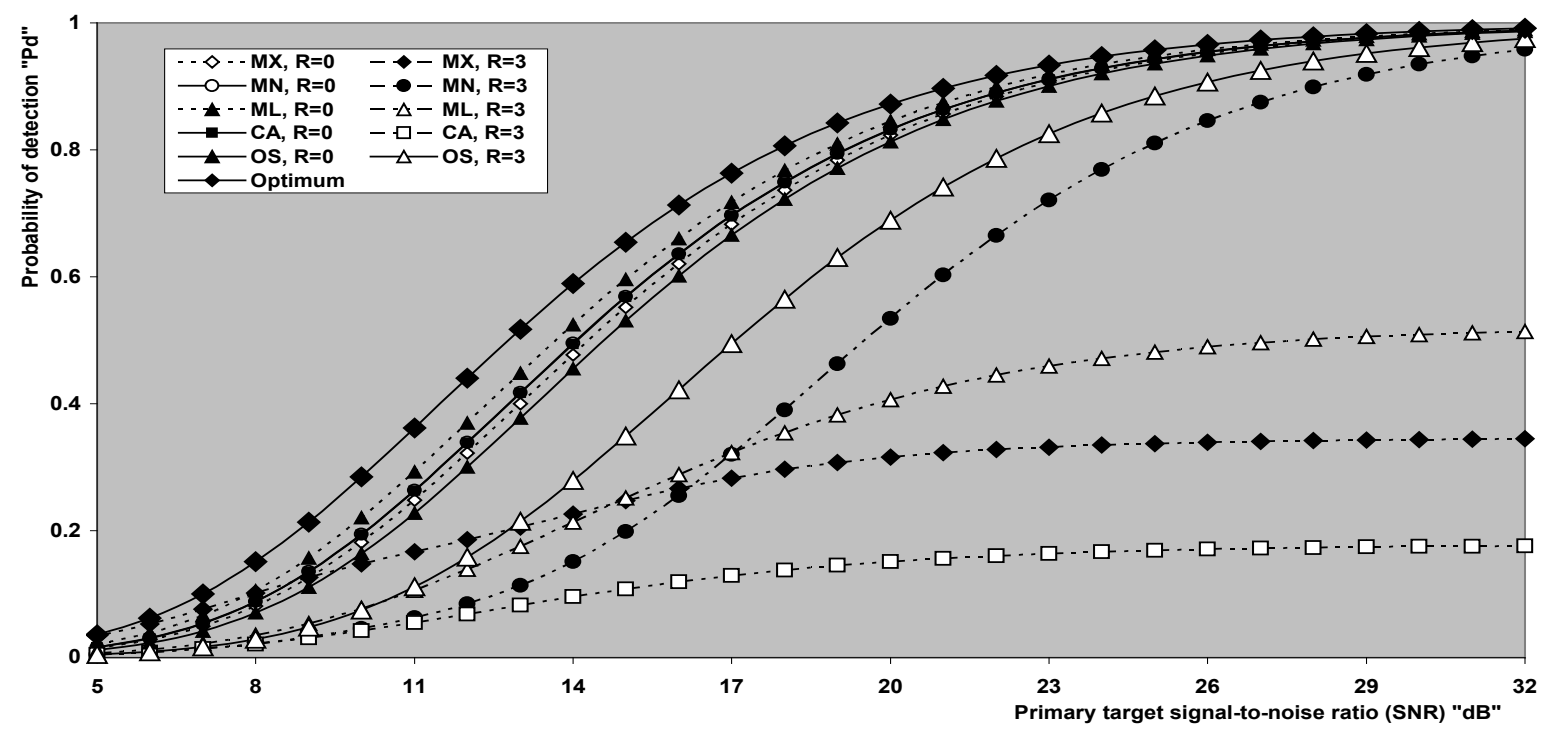

Figure 11. Single pulse detection performance of $C F A R$ processors for $N=24, K=21, R=3$, and $\mathrm{Pfa}=1.1 \mathrm{E}-6$

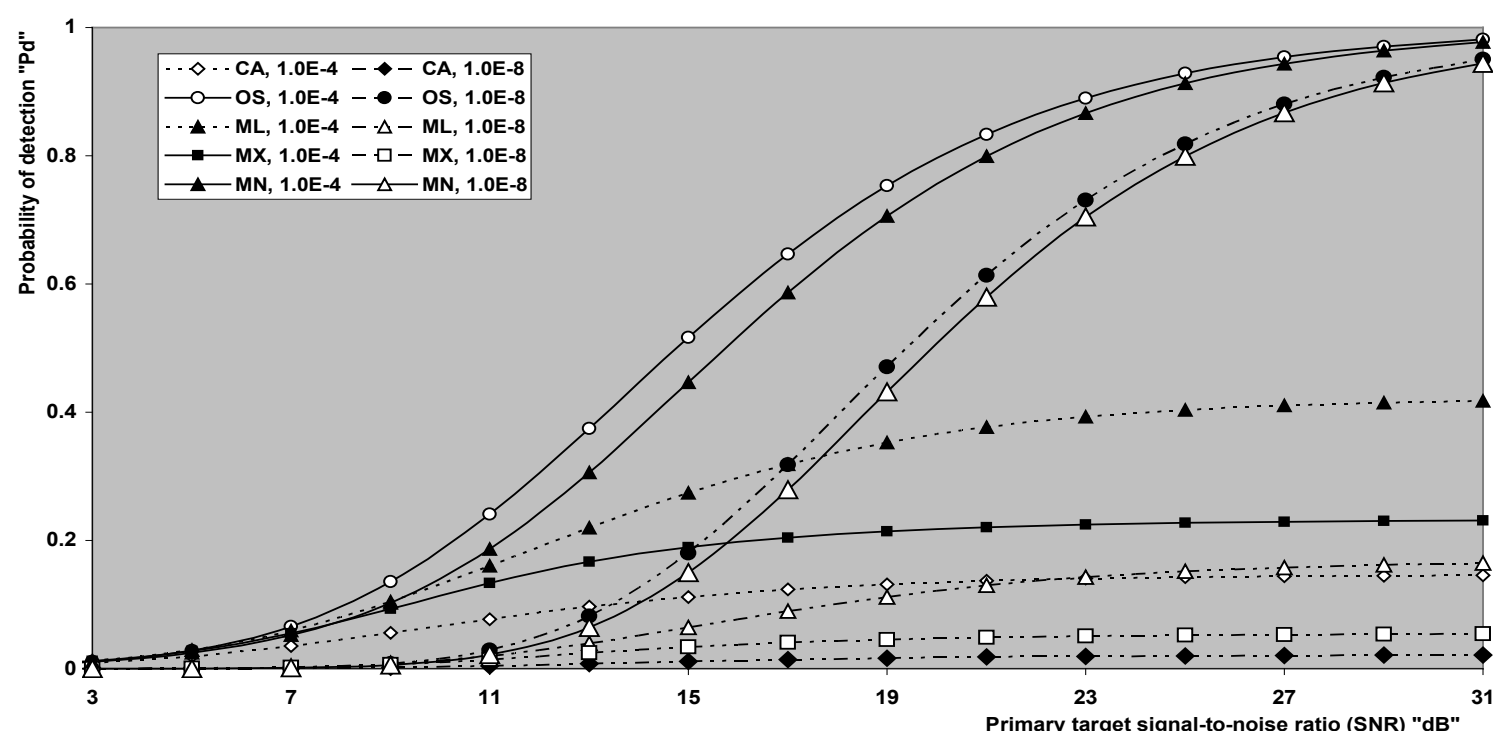

Figure 12. Multiple target detection performance of CFAR processors with fixed value for the ranking order parameter " $K$ " when $N=24, M=1, K=18$, and $\mathrm{R}=5$

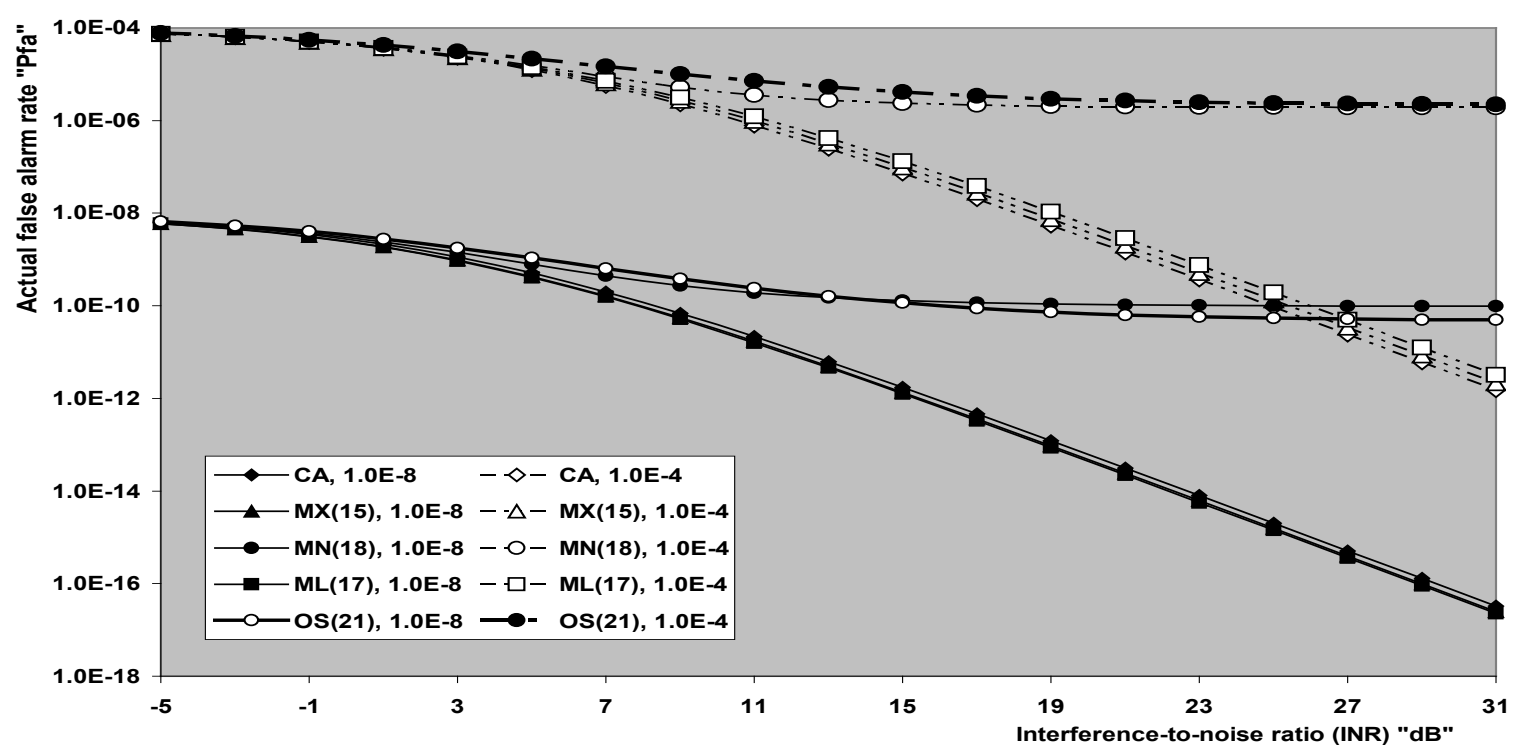

Figure 13. Actual false alarm rate performance of CFAR schemes in target multiplicity environment for $\mathrm{N}=24$, and $\mathrm{R}=3$ 


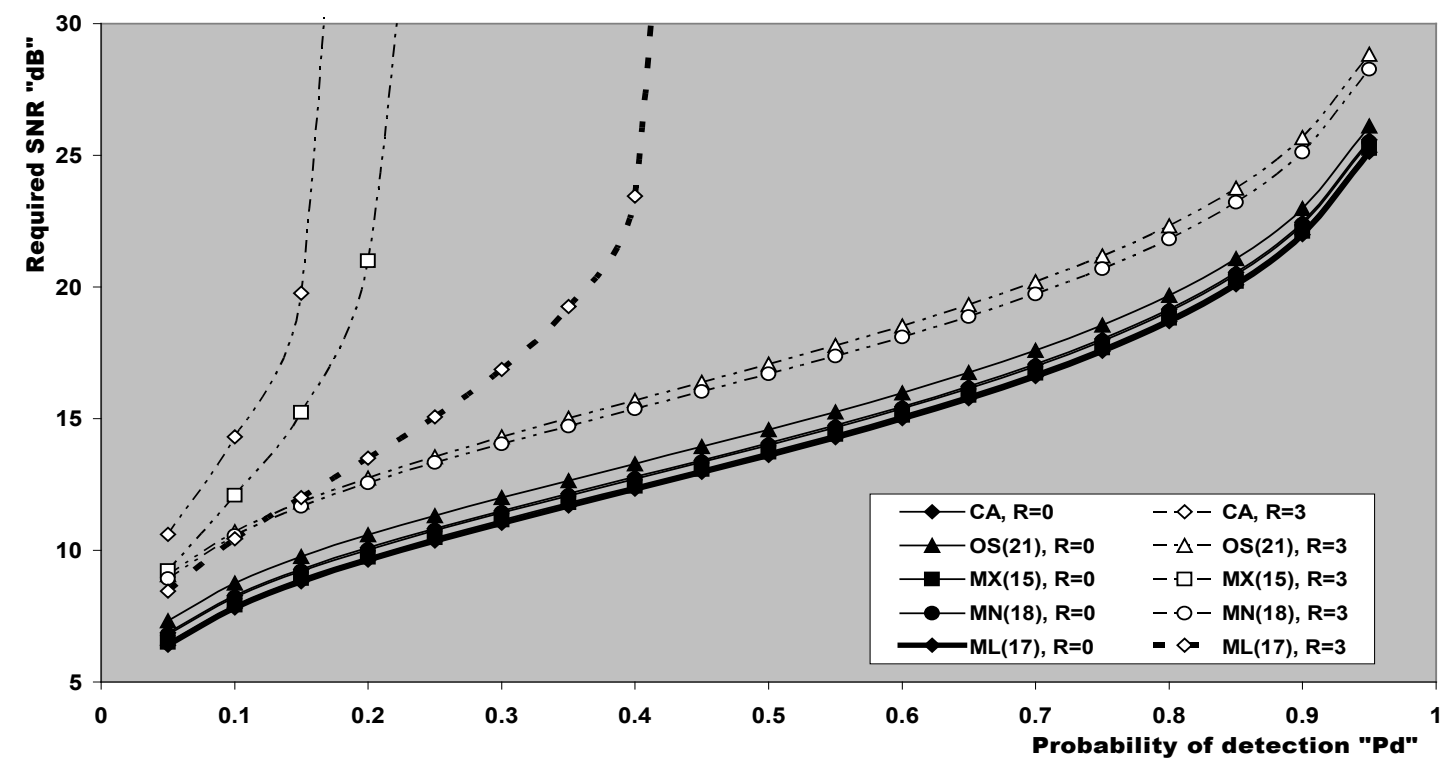

Figure 14. Required SNR to achieve an operating point (1.0E-6, Pd) of CFAR schemes operat ing in multiple target environment with $R=3$, and $N=24$

The presence of multiple targets is another case in studying the non-homogeneous performance of the modified versions. Since the maximum allowable value for the interfering target returns that may exist amongst the candidates of the reference window is $3(\mathrm{R} \leq \mathrm{N}-\mathrm{K})$, the results in Fig.(10) depict the detection performance of the CFAR schemes under consideration for this situation of operating conditions. The displayed curves are obtained for a possible practical application of equal strengths for the outlying as well as the primary target. Two values for the design false alarm rate are taken into account to show the influence of this rate on the behaviour of the CFAR scheme in detecting the radar target. From the variations of the underlined curves, it is obvious that the $\mathrm{CA}$ a lgorith $\mathrm{m}$ has the worst multiple target detection performance and all the developed versions give much better performance than it. However, the ML and MX versions have lower values for the detection probability than the OS procedure. Additionally, the $\mathrm{MN}$ detector is the only one that has a multiple-target detection performance higher than that of the OS scheme. Moreover, the shown results reckoned the well-known identity of the CFAR signal processing that the processor detection performance improves as its design false a larm rate increases. In order to compare the reaction of the CFAR processor to the ideal environment against its behaviour in non-homogeneous situation, Fig.(11) illustrates the detection performance of the under investigated processors in the absence as well as in the presence of interferers for a fixed value of $K$. As a reference for this comparison, the performance of the optimum detector is also included in that figure. The OS(21) has the worst homogeneous performance and the ML version has the highest one, while MN gives the same performance as the CA technique. The MX version has a performance that is higher than the OS scheme but less than the CA processor. On the other hand, the OS gives the highest detection performance when operating in an environment contaminated with three interfering target returns. As expected, the CA has the worst performance. For the modified detectors, the $\mathrm{MN}$ version has a multiple target performance which is close to that of OS, the MX gives the worst, relative to its candidates, and the ML lies in between. These concluded remarks are as sociated with the steady-state behaviour of the underlined processors. Fig.(12) deals with the detection performance of CA and OS techniques along with their extended versions in the presence of spurious targets when there are five contaminated samples. Two design values for the false alarm rate are assumed and the ranking order parameter is held constant at $18(\mathrm{~K}=18)$. The examination of the curves of this figure leads to the same concluded observations as the previous results with minor degradation.

It is of interesting to see to what extent the presence of interferers affect the rate of false alarm of the adaptive scheme of detection. Fig.(13) displays the actual false alarm probability of the underlined processor as a function of the strength of interference when the design rate of false alarm takes the values of $10^{-8}$ and $10^{-4}$. The results of this figure show that the false alarm rate performance of the CA, MX, and ML processors degrades as the strength of the interfering target return (INR) increases. On the other hand, the OS and MN schemes are the only ones that are capable of ma intaining the rate of false a larm constant, especially, when the INR becomes stronger. This result is expected since the largest interfering target returns occupy the top ranked cells and therefore they are not incorporated in the estimation of the background noise power level. In other words, the noise estimate is free of extraneous target returns and therefore it represents the homogeneous background environment. Finally, the value of the SNR, that is required to satisfy a pre-assigned value for the detection probability when the rate of false alarm is held constant, is plotted in Fig.(14). The operating environment is assumed to be free of any interferer as well as in the case where the environment is contaminated with three interferers of the same strength as the target under 
test. In homogeneous situation, the ML(17) requires the minimum SNR to achieve an indicated value for the detection probability. $\mathrm{MX}(15)$ comes in the second class and followed by $\mathrm{MN}(18)$. CA processor comes next and the conventional OS technique requires the highest SNR to achieve the same operating point. On the other hand, the $\mathrm{MN}(18)$ achieves the specified value of detection probability with the minimum SNR when the operating environment is of multiple-targets. In this situation, the OS scheme behaves like MN(18) but with higher values of SNR. The ML(17) achieves only some values for $P_{d}$ and unable to achieve the others since its multiple target detection performance is asymptotically constant at some value which is less than the required ones. The same remark can be said about the behaviour of $\mathrm{MX}(15)$ and $\mathrm{CA}$ procedures.

\section{Conclusions}

In this paper, the detection probability of a radar system that utilizes new versions of adaptive detectors in deciding the presence or absence of fluctuating target in either ideal or non-ideal operating environments was analysed. Three versions of such techniques are processed and closed form expressions are derived for their detection performance. These processors include ML, MX, and MN operations on two separately noise power estimates froma reference set of $\mathrm{N}$ cells: one of them employs CA technique and the other uses OS basis. The analytical results have been used to develop a complete set of performance curves including thresholding constant, ROC's, false alarm rate in clutter edges, detection probability in homogeneous and multiple target situations, required SNR to achieve a prescribed values of $P_{f a}$ and $P_{d}$, and the variation of false alarm rate with the strength of interfering targets that may exist amongst the contents of the estimation set. As expected, the detection performance of the modified versions outweighs that of CA scheme, either in homogeneous or in multiple target environments, for some selected values for the ranking order parameter. From the interference point of view, the considered detectors are partitioned into two families: the CA family and the OS one. The family of CA incorporates ML and MX while that of OS includes MN only. The performance of OS family outweighs that of CA family in non-homogeneous situations. In addition, this family is capable of maintaining a constant rate of false alarm, irrespective to the interference level, in the case where the spurious target returns occupy the top ranked cells and they are within their allowable values. As a final conclusion, the detection performance of the modified versions is related to the ranking order parameter, the target model, the average power of the target, and the environmental operating conditions.

\section{REFERENCES}

[1] Rickard, J. T. \& Dillard, G. M. (1977), “Adaptive detection algorithm for multiple target situations", IEEE Transactions on Aerospace and Electronic Systems, AES-13, (July 1977), pp. 338-343.

[2] Mclane, P. J., Wittke, P. H. \& K-S IP. C. (1982), "Threshold control for automatic detection in radar systems", IEEE Transactions on Aerospace and Electronic Systems, AES-18, (Mar. 1982), pp. 242-248.

[3] P. P. Gandhi and S. A. Kassam (1988), "Analysis of CFAR Processors in nonhomogeneous background", IEEE Transactions on Aerospace and Electronic Systems, AES-24, pp. 427-445, 1988.

[4] El Mashade, M. B. (1994), "M-sweeps detection analysis of cell-averaging CFAR processors in multiple target situations", IEE Proc.-Radar, Sonar Navig., Vol.141, No.2, (April 1994), pp. 103-108.

[5] El Mashade, M. B. (2000), "Detection analysis of CA family of adaptive radar schemes processing $\mathrm{M}$-correlated sweeps in homogeneous and multiple target environments", Signal Processing ,"ELSEVIER”, Vol. 80, (2000), pp. 787-801

[6] Lei Zhao, Weixian Liu, Jeffery S. Fu, Sin Yong Seow and Xin $\mathrm{Wu}$ (2001), 'Two New CFAR Detectors Based on Or-Algorithm and And-Algorithm'; Proceed ings of SCOReD 2001, KL, Malaysia, 2001, pp. 31-34.

[7] El Mashade, M. B. (2002), "Target Multiplicity Performance analysis of radar CFAR detection techniques for partially correlated chi-square targets", Int. J. Electron. Commun. (AEÜ), Vol.56, No.2, (April 2002), pp. 84-98.

[8] Biao Chen, Pramod K. Varshney \& James H. Michels (2003), "Adaptive CFAR Detection for Clutter-Edge Heterogen eity Using Bay esian Inference", IEEE Transactions on Aerospace and Electronic Systems Vol. 39, No. 4, October 2003, pp.1462-1470.

[9] El Mashade, M. B. (2005), "M-Sweeps exact performance analysis of OS modified versions in nonhomogeneous environments", IEICE Trans. Commun., Vol.E88-B, No.7, (July 2005), pp. 2918-2927

[10] El Mashade, M. B. (2006), “Analysis of cell-averaging based detectors for $\chi^{2}$ fluctuating targets in multitarget environments", Journal of Electronics (China) 23 (6) (November 2006), pp. 853-863.

[11] Simon Haykin (2007), "Adaptive Radar Signal Processing”, A John Wiley \& Sons, Inc., Publication

[12] El Mashade, M. B. (2008), "Performance analysis of OS structure of CFAR detectors in fluctuating targets environments", Progress in Electromagnetics Research (PIER) C2 (2008) 127-158.

[13] El Mashade, M. B. (2011), “Analysis of adaptive detection of moderately fluctuating radar targets in target multiplicity environments", Journal of the Franklin Institute 348 (2011) 941-972 